\title{
UNDER STOCHASTIC DOMINANCE CHOQUET-EXPECTED UTILITY AND ANTICIPATED UTILITY ARE IDENTICAL
}

\begin{abstract}
The aim of this paper is to convince the reader that Choquet-expected utility, as initiated by Schmeidler $(1982,1989)$ for decision making under uncertainty, when formulated for decision making under risk naturally leads to anticipated utility, as initiated by Quiggin/Yaari. Thus the two generalizations of expected utility in fact are one.
\end{abstract}

Keywords: Nonadditive probabilities, decision making under risk, decision making under uncertainty, prospect theory, Choquet-expected utility, anticipated utility, rank-dependent utility, cumulative utility, comonotonicity.

\section{HISTORY, MOTIVATION AND PREVIEW}

In the beginning of the eighties Schmeidler (1982; published 1989) and Quiggin/Yaari (published 1982/1987) devised new generalizations of expected utility. The new idea of both of these generalizations was to deal with probabilities in a nonadditive way. Many previous trials to use nonadditive probabilities (e.g. in Edwards, 1954, and in Kahneman and Tversky, 1979; these authors themselves did not use an interpretation of nonadditive probabilities) adopted a way to integrate with respect to nonadditive probabilities, seemingly dual to expected utility, but at closer study unsound. Kahneman and Tversky themselves already pointed out the major problem in their way of integration; further comments are given in Quiggin (1982), and illustrations are given in Wakker (1989c). The problem had already been known to $\mathrm{L}$. J. Savage. The main contribution of Schmeidler and Quiggin/Yaari was to find a sound way to integrate with respect to nonadditive probabilities, a way which turned out to have been found already by the mathematician Choquet (1953/4).

It was immediately understood that the ways of integration of Schmeidler and Quiggin/Yaari were related, see for instance Yaari (1987, p. 114). Nevertheless the data equivalence of the two approaches for decision making under risk (DMUR), as pointed out in 
this paper, has not been observed before, to the best of our knowledge. A possible explanation may be the following. In Schmeidler's approach, which we propose to call the Choquet-expected utility $(C E U)$ approach, the 'subjective' probabilities (not given $a$ priori) are dealt with in a new, nonadditive, way. Also there occur objective ('known') probabilities, but it is essential for Schmeidler's proof, and also in accordance with his interpretations, that these be dealt with in the (traditional) additive way. The crucial new idea in Quiggin/Yaari's set-up, to the contrary, is that objective ('known') probabilities be dealt with in a nonadditive way.

Nevertheless the approach of Quiggin/Yaari, the anticipated utility $(A U)$ approach, is the natural version of the CEU approach when adapted to DMUR, as we shall argue. To do this, firstly an alternative derivation of the CEU approach must be established, one which does not need the auxiliary tool of objective probabilities to be dealt with in an additive way. Such an alternative derivation has been obtained in Gilboa (1987), Wakker (1989a, b), and Nakamura (1990). Once the possibility of such an alternative derivation has been accepted, knowledge of its details is not needed for the identification of CEU and AU. Hence this paper does not repeat those details.

Secondly (see Section 3), we have to show that DMUR, the context to which $\mathrm{AU}$ applies, can be considered a special case of decision making under uncertainty (DMUU), the context to which CEU applies. We shall only show the formal relatedness, and not enter conceptual discussions. The work of Section 3 has use on its own, in elucidating the risk/uncertainty dichotomy as introduced by Knight (1921), and in giving an 'algorithm' to translate results from DMUR to DMUU and vice versa. Section 3 can be read independently of Subsections 2.2 and 2.3 .

Now CEU can directly be applied to DMUR. Then, thirdly (see Section 4) and finally, we show equivalence of the following two approaches to DMUR:

- AU with stochastic dominance.

- CEU with stochastic dominance.

The obtained identification enables the application of techniques, 
developed before for $\mathrm{CEU}$, to $\mathrm{AU}$. Thus results for $\mathrm{AU}$ can be generalized; this will be elaborated in Wakker (1990). We derive the present result in full generality, without imposing restrictions upon the state space, probabilities, probability transformations, consequences, utilities, or whatever. To do this, in Definition 5 the natural version of stochastic dominance for general consequence spaces will be given, generalizing the traditional stochastic dominance that has been formulated for monetary consequences and increasing utility.

Proofs are given in the Appendix. Let us finally refer to Fishburn (1988), giving a survey of $\mathrm{CEU}, \mathrm{AU}$, and many other deviations from expected utility.

\section{CEU FOR DMUU}

In this section we sketch the CEU approach to DMUU, as initiated by Schmeidler $(1982,1989)$. For easy accessibility we shall not introduce measure-theoretic structure; the latter can be introduced exactly as in Schmeidler $(1982,1989)$ (supplemented in Wakker, 1989e).

\subsection{Elementary Definitions of Decision Making Under Uncertainty}

In decision making under uncertainty (DMUU) the lack of information of the decision maker is modeled through a set $S$, the set of states (of nature). Exactly one state is the 'true state', the other states are not true. Subsets of $S$ are events. A decision maker is uncertain about which state of nature is true, and has not any influence on the truth of the states. As an example one may think of a horse race that will take place. Every horse is identified with a state; $s \in S$ designates the 'state' that horse $s$ will win.

$\mathscr{C}$ denotes the set of consequences. The set $\mathscr{F}$ is the set of all acts, i.e., functions from $S$ to $\mathscr{C}$. If a decision maker chooses an act $f$, this results in consequence $f(s)$ for (him or) her, where $s$ is the true state of nature. For instance, acts may designate bets for money on horses. Since the decision maker is uncertain about which horse will win, (he or) she is uncertain which amount of money will result from a bet. By $\geq$ we denote the preference relation of the decision maker on $\mathscr{F}$. As usual, we write $f>g$ if $f \geq g$ and not $g \geq f, f \leq g$ if $g \geq f, f<g$ if 
$g>f$, and $f \simeq g$ if $f \geq g$ and $g \geq f$. We call $\geq$ a weak order if it is complete ( $f \geq g$ or $g \geq f$ for all $f, g$ ) and transitive. Further $\geq$ is trivial if $f \geq g$ for all $f, g$. A function $V: \mathscr{F} \rightarrow \Re$ represents $\geq$ if $[f \geq$ $g] \Leftrightarrow[V(f) \geqslant V(g)]$. (The term utility function is reserved in this paper for the function $U$ in the sequel; the expectation of $U$ will represent $\geq$.) We shall often identify consequences with the associated constant acts. Thus, for $\alpha, \beta \in \mathscr{C}$, we may write $\alpha \geq \beta$, etc.

\subsection{The Choquet-Integral and $C E U$}

A function $v: 2^{S} \rightarrow[0,1]$ is a capacity ('nonadditive probability') if $v(\emptyset)=0, v(S)=1$, and $v$ is monotonic w.r.t. set-inclusion, i.e., $A \supset$ $B \Rightarrow v(A) \geqslant v(B)$. Further $v$ is a probability measure if it is a capacity that is additive, i.e., $v(A \cup B)=v(A)+v(B)$ for all disjoint $A, B$. In literature often a continuity condition is imposed on capacities, requiring for any sequence of events $\left(A_{1}, A_{2}, \ldots\right)$ :

$$
\left[\forall j: A_{j+1} \subset A_{j}, \bigcap_{j=1}^{\infty} A_{j}=A\right] \Rightarrow\left[\lim _{j \rightarrow \infty} v\left(A_{j}\right)=v(A)\right],
$$

and requiring the same with $\supset, \cup$ instead of $\subset, \cap$. In this paper the continuity restriction is not imposed. For probability measures the continuity condition is known to be equivalent to countable additivity, i.e., for any countable disjoint sequence of events the probability of the union of these events is the sum of the probabilities. Whenever we require countable additivity of a probability measure we will make it explicit.

For a capacity $v$, and a function $\phi: S \rightarrow \Re$, the Choquet-integral of $\phi$ (with respect to $v$ ), denoted $\int_{S} \phi \mathrm{d} v$, or $\int \phi \mathrm{d} v$, is

$$
\begin{aligned}
& \int_{0}^{\infty} v(\{s \in S: \phi(s) \geqslant \tau\}) \mathrm{d} \tau \\
& \quad+\int_{-\infty}^{0}[v(\{s \in S: \phi(s) \geqslant \tau\})-1] \mathrm{d} \tau .
\end{aligned}
$$

For probability measures the Choquet-integral is identical to the usual integral. Elucidations and illustrations for the Choquet-integral are given in Wakker (1989b, Section VI.2). 
DEFINITION 1. We say that Choquet-expected utility (CEU) applies if there exist a utility function $U: \mathscr{C} \rightarrow \mathfrak{A}$ and a capacity $v$ on $S$, so that $f \mapsto \int_{S}(U \circ f) \mathrm{d} v$ represents $\geq$. The integral is the Choquet-expected utility (CEU) of $f$.

\subsection{Transforming Additive Probabilities}

One way to obtain a capacity is to take an additive probability measure $P$ on $S$, a nondecreasing function $\varphi:[0,1] \rightarrow[0,1]$ with $\varphi(0)=0$, $\varphi(1)=1$, and then take as capacity the 'distorted probability' $v:=\varphi \circ P$. Obviously the probability measure $P$ may simply be a mathematical device, without any interpretation associated with it. Two other extreme cases are, firstly, the case of DMUU, where $P$ is not given and must be derived from other sources, and secondly, the case of DMUR, where $P$ is a 'given objective' probability measure, known in advance. This second case will be considered in Section 4. Let us now comment shortly on the first case.

Under CEU one may wonder whether there exists a probability measure $P$ so that $v=\varphi^{\circ} P$, with $\varphi$ nondecreasing. This is the case if and only if there exists a probability measure $P$ so that, for $\geq$ ' on $S$ defined by $A \geq^{\prime} B \Leftrightarrow v(A) \geqslant v(B)$, we have $P(A) \geqslant P(B) \Rightarrow A \geq^{\prime} B$. Questions about the existence of such a probability measure $P$ are studied in 'comparative probability theory', see Wakker (1981, supplementing Savage, 1954), Gilboa (1985, explicitly indicating the significance for AU), or Fishburn (1986, giving a survey). See also Luce (1988, Section 6). The five-point example in Kraft, Pratt, and Seidenberg (1959) can be used to show that there exist, even for a finite state space, capacities which cannot be obtained as nondecreasing transforms of an additive probability, even so when the capacities satisfy a kind of 'ordinal additivity'.

Section 4 will introduce AU, and will show that it is a special case of CEU, so that it is not more general. The above observation shows, in addition, that CEU is strictly more general.

\section{FROM DMUU TO DMUR AND VICE VERSA}

In Subsection 2.1 we sketched the approach of DMUU. In the usual approach to DMUR, which we shall also call DMUR without states, 
one takes as point of departure a consequence set $\mathscr{C}$, a collection of countably additive probability distributions over $\mathscr{C}$, and a preference relation over these probability distributions.

As a preparation for showing the relatedness of $\mathrm{AU}$ and $\mathrm{CEU}$, this elementary section shows the way to consider DMUR as a special case of DMUU, in the sense that DMUU requires less structure so can be applied more generally. The main step is carried out in Subsection 3.2, showing that in DMUR it is no restriction to assume an 'underlying' state space. In Subsection 3.1 we start from the general model of DMUU, and show which restrictive assumptions must be added to end up in a case of DMUR. Subsection 3.2 then shows that all cases of DMUR can be thought to have been obtained like that.

\subsection{From DMUU to DMUR}

$(D M U U)$ Firstly, we start from the general set-up of DMUU as described in Subsection 2.1, with as primitives the state space $S$, the consequence set $\mathscr{C}$, the acts, and the preference relation $\geq$ over the acts.

(Intermediate set-up) Secondly, we add the assumption that at the outset an objective probability measure $P$ on $S$ is given; it is custom to assume, and so shall we do, that this objective probability measure is countably additive. The set-up now obtained will be called the intermediate set-up. Any act $f: S \rightarrow \mathscr{C}$ induces a countably additive probability distribution over the consequences, assigning to every subset of the consequence set the probability of its inverse under the act. In the intermediate set-up the probability distribution can be without any relevance to the decision maker.

(DMUR with states) Thirdly, we add the restrictive assumption characteristic for DMUR. It states that all relevant information of an act is contained in the probability distribution which the act induces on the consequences. Its formal statement is as follows:

ASSUMPTION 2 [DMUR]. If two acts induce the same probability distribution over consequence, then they are $(\simeq-)$ equivalent.

Yaari (1987) called this assumption 'neutrality', Fishburn (1988) called it the 'reduction principle'. It will be implied by the stronger and 
natural condition of stochastic dominance as we shall formulate it in the sequel. We call the set-up now obtained DMUR with states. In it, (preferences on) acts and probability distributions are equivalent; they will often be identified and interchanged.

(DMUR without states) Fourthly and finally, if all acts which induce the same probability distribution over consequences are equivalent, then we may describe any act by the induced probability distribution, forget about the state space, the acts, and $P$, and use the naturally induced preference relation on the probability distributions over consequences. This is in accord with a tradition in mathematical statistics and probability theory. There one often works with probability distributions, having in mind that these are probability distributions of random variables (= acts) defined on a probability space, but leaving the probability space and the random variables unmentioned, simply because these are needed nowhere in the analysis. What has resulted is the usual approach of DMUR, without states. In literature there is usually the further restrictive assumption that all probability distributions over (a $\sigma$-field over) $\mathscr{C}$ must be available. To achieve that, we must add the assumption that $(S, P)$ is 'rich enough' to generate all these. The next subsection will give further comments.

\subsection{From DMUR to DMUU}

To claim that DMUR is truly a special case of DMUU, we must show that each case of DMUR can be the result of the procedure sketched in the previous subsection. I.e., starting from the usual set-up of DMUR (without states), with a consequence set $\mathscr{C}$ and countably additive probability distributions over $\mathscr{C}$, we must show that we can always construct a state space $S$ with a probability distribution $P$ on $S$, generating all the considered probability distributions. This is shown in the next paragraph, is somewhat technical, and may be taken for granted.

One can for instance assume that all considered probability distributions are independent, and take as probability space a Cartesian product of dimension as large as the cardinality of the set of considered probability distributions, with all coordinate sets equal to $\mathscr{C}$. This procedure can also be adopted if the probability measures are not countably additive, and $\mathscr{C}$ is general. In DMUR, where choices 
between probability distributions are to be made by a decision maker, joint distributions of different probability distributions over $\mathscr{C}$ are not considered, they are irrelevant. Hence the above procedure can be considered a trivial application of a theorem of Kolmogorov, see Feller (1966, Theorem IV.6.1). Usually simpler spaces will suffice. For instance, for the case $\mathscr{C} \subset \mathfrak{R}$, the state space $[0,1]$ endowed with the uniform distribution will suffice to generate all countably additive probability distributions over $\mathfrak{R}$, through generalized 'inverses' of distribution functions.

It has now also been established that there is no essential difference between DMUR with or without states. We may always assume the states to be given, and so we shall do.

\section{AU AS SPECIAL CASE OF CEU}

In this section we introduce $\mathrm{AU}$, and show how to derive it from CEU.

\section{1. $A U$}

We consider the context of DMUR with states. The integral below is a Choquet-integral, with respect to the capacity $\varphi \circ P$.

DEFINITION 3. Anticipated utility $(A U)$ applies when there exist a utility function $U: \mathscr{C} \rightarrow \mathfrak{\Re}$ and a nondecreasing transformation $\varphi:[0,1] \rightarrow[0,1]$ with $\varphi(0)=0, \varphi(1)=1$, so that (with $P_{f}$ the probability distribution over $\mathscr{C}$ induced by an act $f) \geq$ is represented by

$$
P_{f} \mapsto \int_{S}(U \circ f) \mathrm{d} \varphi \circ P
$$

The integral is the anticipated utility $(A U)$ of the act $f$, or of the distribution $P_{f}$.

As the essential new idea of AU we take the fact that first the probability measure $P$ is transformed nondecreasingly before expectation is calculated. Other conditions met in literature, such as continuity of $\varphi$ and linearity of $U$ as in Yaari (1987), or continuity of $\varphi$ and the 
equality $\varphi\left(\frac{1}{2}\right)=\frac{1}{2}$ (and possibly some unmentioned continuity of $U$ ) as in Quiggin (1982), or boundedness of $U$ and convex-rangedness of $\varphi \circ P$ as in Gilboa (1987), or continuity of $U$ as in the author's papers, or continuity and strict increasingness of $U$ and $\varphi$ as in Chew (1989), or 'solvability' as in Nakamura (1990), to our view are not crucial aspects of AU, and are only used to simplify analyses.

Several alternative terms for AU are used in literature. Chew, Karni and Safra (1987) use the term 'expected utility with rank dependent probabilities'. Yaari (1987) uses the term 'dual theory'; this seems most suited for the special case of linear utility, as considered in his paper. AU theory in full generality is a generalization of expected utility, rather than a dual. Also the appealing term 'cumulative utility' has occurred.

The following equivalent formulation is suited for DMUR without states, because no states or acts are used. For simplicity we assume that $U$ is nonnegative. Let $G_{U \circ f}$ be the decumulative distribution function of $U \circ f$, i.e., $G_{U \circ f}: \tau \mapsto P([U \circ f \geqslant \tau])$. The representing function in (2) can be seen to be equal to:

$$
P_{f} \mapsto \int_{[0, \infty[} \tau \mathrm{d}\left(-\varphi \circ G_{U \circ f}(\tau)\right)
$$

This formulation shows that different acts which induce the same probability distribution over $\mathscr{C}$ are valued the same, as should be for DMUR. One can consider the above integral to be a usual (expectedutility-)integral, not of the distribution given by $G_{U \circ f}$, but of the 'transformed distribution' given by $\varphi \circ G_{U_{\circ} f}$. Right-continuity of $\varphi$ will guarantee that $\varphi \circ G_{U \circ f}$ indeed is the decumulative distribution function of a countably additive distribution; continuity of $\varphi$ will be characterized in Wakker (1990). We prefer not to require continuity of $\varphi$ in the general definition of $\mathrm{AU}$, because we want to be able to include for instance maxmin behavior, and other kinds of discontinuities at probabilities 0 or 1 .

We could write (3) alternatively by deleting the minus sign and replacing $\varphi \circ G_{U \circ f}$ by $\psi \circ F_{U \circ f}$, with $\psi: \tau \mapsto 1-\varphi(1-\tau)$, and $F_{U \circ f}$ the usual cumulative distribution function of $U \circ f$, i.e., $F_{U \circ f}: \tau \mapsto$ $P([U \circ f \leqslant \tau])$. 


\subsection{Applying CEU to DMUR}

Let us start from the 'intermediate set-up' described in Subsection 3.1, with given $P$ but without Assumption 2. This is a special case of DMUU, and nothing prevents us from applying CEU. The objective probability measure $P$, while present, simply does not have to be used. The following lemma shows when it is used after all.

LEMMA 4. Let $\geq$ be nontrivial, let the intermediate set-up apply, and let CEU apply. Then there exists a transformation $\varphi:[0,1] \rightarrow[0,1]$ so that $v=\varphi \circ P$ if and only if Assumption 2 applies, i.e., if and only if DMUR with states applies.

The above lemma does not yet give AU because the transformation $\varphi$ does not have to be nondecreasing. For example, with $\mathscr{C}$ a nondegenerate interval, $U$ identity, $S=\left\{s_{1}, s_{2}\right\}$, and $P\left(s_{1}\right)>P\left(s_{2}\right)>0$, we are still free to choose $\varphi\left(P\left(s_{1}\right)\right)<\varphi\left(P\left(s_{2}\right)\right)$. However, if the state space $S$ is so rich that for every $P(A)>P(B)$ we can find a $B^{\prime}$ so that $P\left(B^{\prime}\right)=P(B)$ and $B^{\prime} \subset A$, then because of monotonicity of capacities $\varphi(P(A)) \geqslant \varphi\left(P\left(B^{\prime}\right)\right)=\varphi(P(B))$ follows. The mentioned richness holds, under countable additivity of $P$, if and only if either the state space is atomless, or it consists of a finite number of equally-probable atoms. We will not derive AU from the richness-condition in the way as just suggested, but rather from the natural condition of stochastic dominance. Stochastic dominance is usually formulated for the case where consequences are real numbers and (utility/)preferences are increasing. The condition below is the natural generalization to general consequences. Also it is the natural generalization to acts instead of distributions.

DEFINITION 5. We say for acts $f, g$ that $f$ (weakly, first-order) stochastically dominates $g$ if

$$
[\forall \alpha \in \mathscr{C}: P(f(s) \geq \alpha) \geqslant P(g(s) \geq \alpha)] .
$$

We say $\geq$ satisfies (monotonicity w.r.t. weak first-order) stochastic dominance if $f \geq g$ whenever $f$ stochastically dominates $g$. 
Obviously, if $f$ and $g$ induce the same probability distribution over the consequences, then they stochastically dominate each other. By stochastic dominance of $\geq, f$ and $g$ then must be equivalent. So we have:

$$
\text { Stochastic dominance of } \geq \text { implies Assumption } 2 \text {. }
$$

I.e., under stochastic dominance DMUR applies. It may be argued that our version of stochastic dominance for acts contains two separate ideas, firstly, the idea of Assumption 2, secondly, the idea of stochastic dominance for distributions over consequences.

LEMMA 6. Let $\geq$ be nontrivial. Let the intermediate set-up apply, and let CEU apply. Then there exists a nondecreasing transformation $\varphi:[0,1] \rightarrow[0,1]$ so that $v=\varphi \circ P$, if and only if stochastic dominance is satisfied.

The existence of $\varphi$ in the above lemma is exactly what defines AU. Since by definition $\mathrm{AU}$ is a special case of CEU, the following theorem results:

THEOREM 7. Let $\geq$ be nontrivial. Let the intermediate set-up apply, and let stochastic dominance hold. Then CEU applies if and only if $A U$ applies.

\section{CONCLUSION}

We have given a general procedure for 'translating' results from decision making under uncertainty into results for decision making under risk. This suggests that decision making under uncertainty is the more basic of the two set-ups. By means of the procedure we have shown that two generalizations of expected utility, introduced independently for different contexts, at closer study in fact are one. We rephrase, taking the noncontroversial stochastic dominance for granted,

Choquet-expected utility, when applied to DMUR, is identical to Anticipated utility. 


\section{ACKNOWLEDGEMENT}

The research has been made possible by a fellowship of the Royal Netherlands Academy of Arts and Sciences, and a fellowship of the Netherlands Organization for Scientific Research.

\section{APPENDIX; PROOFS}

PROOF of Lemma 4. First suppose Assumption 2 holds. We prove that $v=\varphi \circ P$ for some transformation $\varphi$. It suffices to show that for any events $A, B, P(A)=P(B) \Rightarrow v(A)=v(B)$. So let $P(A)=P(B)$. Since $\geq$ is nontrivial, the utility function $U$ used in CEU is not constant. So let $U(\alpha)>U(\beta)$. According to Assumption 2 we have, with $\alpha 1_{A}+\beta 1_{A^{c}}$ denoting the act assigning $\alpha$ to $A$ and $\beta$ to $A^{c}$, $\alpha 1_{A}+\beta 1_{A^{c}} \simeq \alpha 1_{B}+\beta 1_{B^{c}}$. Substituting CEU's gives $v(A) U(\alpha)+(1-$ $v(A)) U(\beta)=v(B) U(\alpha)+(1-v(B)) U(\beta)$. This implies $v(A)=v(B)$.

Conversely, suppose a $\varphi$ as in the lemma exists. We derive Assumption 2. Let acts $f$ and $g$ induce the same probability distribution over consequences. Then each of these acts has the same CEU since in the Choquet-integrals of $f \circ U$ respectively $g \circ U$ we find the same integrands.

PROOF of Lemma 6. First suppose $\varphi$ exists and is nondecreasing. To derive stochastic dominance, let $f$ stochastically dominate $g$. By nondecreasingness of $\varphi, \varphi(P(U \circ f \geqslant \tau)) \geqslant \varphi(P(U \circ g \geqslant \tau))$. Substituting this in the Choquet-integrals of $U \circ f$ and $U \circ \dot{g}$ shows that $f \geq g$.

Next we assume stochastic dominance holds, and derive (existence, which could also be derived from Formula 4 and Lemma 4 and) nondecreasingness of $\varphi$. It can be seen that it is sufficient to show that for any events $A, B, P(A) \geqslant P(B) \Rightarrow v(A) \geqslant v(B)$. So let $P(A) \geqslant$ $P(B)$. Since $\geq$ is nontrivial, the utility function $U$ used in CEU is not constant. So let $U(\alpha)>U(\beta)$. By stochastic dominance we have $\alpha 1_{A}+\beta 1_{A^{c}} \geq \alpha 1_{B}+\beta 1_{B^{c}}$. Substituting CEU's gives $v(A) U(\alpha)+$ $(1-v(A)) U(\beta) \geqslant v(B) U(\alpha)+(1-v(B)) U(\beta)$. This implies $v(A) \geqslant$ $v(B)$. 


\section{REFERENCES}

Chew, S. H.: 1989, 'An Axiomatic Generalization of the Quasilinear Mean and Gini Mean with Application to Decision Theory', Johns Hopkins University and Tulane University; rewritten version of Chew, S. H. (1985), "An Axiomatization of the Rank-Dependent Quasilinear Mean Generalizing the Gini Mean and the Quasilinear Mean', Economics Working Paper \#156, Johns Hopkins University.

Chew, S. H., Karni, E., and Safra, Z.: 1987, 'Risk Aversion in the Theory of Expected Utility with Rank Dependent Probabilities', Journal of Economic Theory 12, 370--381.

Choquet, G.: 1953-4, 'Theory of Capacities', Annales de l'Institut Fourier (Grenoble), 131-295.

Edwards, W.: 1954, 'The Theory of Decision Making', Psychological Bulletin 51, 380-417.

Feller, W.: 1966, An Introduction to Probability Theory, Vol. II, Wiley, New York.

Fishburn, P. C.: 1986, 'The Axioms of Subjective Probability', Statistical Science 1, 335-358.

Fishburn, P. C.: 1988, Nonlinear Preference and Utility Theory, Johns Hopkins University Press, Baltimore.

Gilboa, I.: 1985, 'Subjective Distortions of Probabilities and Non-Additive Probabilities', Working paper 18-85, Foerder Institute for Economic Research, Tel-Aviv University, Ramat Aviv, Israel.

Gilboa, I.: 1987, 'Expected Utility with Purely Subjective Non-Additive Probabilities', Journal of Mathematical Economics 16, 65-88.

Kahneman, D. and Tversky, A.: 1979, 'Prospect Theory: An Analysis of Decision under Risk', Econometrica 47, 263-291.

Knight, F. H.: 1921, Risk, Uncertainty, and Profit, Houghton Mifflin, New York.

Kraft, C. H., Pratt, J. W., and Seidenberg, A.: 1959, 'Intuitive Probability on Finite Sets', Annals of Mathematical Statistics 30, 408-419.

Luce, R. D.: 1988, 'Rank-Dependent, Subjective Expected-Utility Representations', Journal of Risk and Uncertainty 1, 305-332.

Nakamura, Y.: 1990, 'Subjective Expected Utility with Non-Additive Probabilities on Finite State Space', University of Tsukuba, Tsukuba, Ibaraki, Japan.

Quiggin, J.: 1982, 'A Theory of Anticipated Utility', Journal of Economic Behaviour and Organization 3, 323-343.

Savage, L. J.: 1954, The Foundations of Statistics, Wiley, New York. (Second edition 1972, Dover, New York.)

Schmeidler, D.: 1982, 1989, 'Subjective Probability and Expected Utility without Additivity', Econometrica 57 (1989), 571-587; first version 1982.

Wakker, P. P.: 1981, 'Agreeing Probability Measures for Comparative Probability Structures', The Annals of Statistics 9, 658-662.

Wakker, P. P.: 1989a, 'Continuous Subjective Expected Utility with Nonadditive Probabilities', Journal of Mathematical Economics 18, 1-27.

Wakker, P. P.: 1989b, Additive Representations of Preferences, A New Foundation of Decision Analysis, Kluwer (Academic Publishers), Dordrecht.

Wakker, P. P.: 1989c, 'Transforming Probabilities without Violating Stochastic Dominance', in E. E. Ch. I. Roskam (ed.), Mathematical Psychology in Progress, Springer, Berlin, 29-48. 
Wakker, P. P.: 1989d, 'A Behavioral Foundation for Fuzzy Measures', Fuzzy Sets and Systems, forthcoming.

Wakker, P. P.: 1989e, 'From Finite- to Infinite-Dimensional Integral Representations; Unbounded Utility for Savage (1954) and Others', Duke University, Fuqua School of Business, working paper 8928 .

Wakker, P. P.: 1990, In preparation.

Yaari, M. E.: 1987, 'The Dual Theory of Choice under Risk', Econometrica 55, 95-115.

University of Nijmegen,

Nijmegen Institute for Cognition research and

Information technology (NICI),

Nijmegen, The Netherlands. 